\title{
Control of Nonlinear Teleoperation Systems Subject to Disturbances and Variable Time Delays
}

\author{
Alireza Mohammadi, Mahdi Tavakoli and Horacio J. Marquez
}

\begin{abstract}
Instability and poor performance are two wellknown problems encountered in bilateral teleoperation over a communication channel with variable time delays, where force feedback from the slave side is provided to the master side. When unknown disturbances or external forces act on the master and/or the slave manipulators, the teleoperation system will be even more prone to stability and performance degradation. By adopting a Lyapunov approach, we present a novel nonlinear disturbance observer based control scheme for teleoperation systems that are subject to variable time delays and disturbances. Lumping the effects of dynamic uncertainties, unknown forces/torques exerted by the human operator and the remote environment, and external disturbances into a single disturbance term enables us to use a disturbance observer and suppress these disturbances in order to alleviate their adverse effects on the teleoperation system stability and performance. The proposed disturbance observer based control laws guarantee asymptotic disturbance tracking, asymptotic position tracking, and stability of teleoperation system in both constrained and free motions. Experimental results are presented to verify the effectiveness of the proposed approach.
\end{abstract}

\section{INTRODUCTION}

A teleoperation system consists of a master (local) manipulator (also known as the user interface), a communication channel, and a slave (remote) manipulator. Different types of information such as force, position, and visual/auditory data are exchanged between the remote and the local sides via the communication channel. If force feedback from the slave side to the master side is present, then the system is called a bilateral teleoperation system to distinguish it from a unilateral teleoperation system, in which no force is reflected to the user. A bilateral teleoperation system is said to be transparent if the slave manipulator accurately follows the position of the master manipulator and the master manipulator faithfully reflects the slave-environment contact force to the human operator. Haptic force feedback has been shown to enhance the human operator's performance in teleoperated tasks in terms of task success rate and economy of exerting forces. Also, in teleoperated assembly tasks, it has been observed that the presence of haptic force feedback can

This work was supported by the Natural Sciences and Engineering Research Council of Canada (NSERC). This paper represents some results from the thesis work done by A. Mohammadi in the course for the degree of Master of Science from the University of Alberta. The thesis was supervised by Dr. M. Tavakoli and Dr. H. J. Marquez.

A. Mohammadi was with the Department of Electrical \& Computer Engineering, University of Alberta, Edmonton, AB T6G 2V4 Canada. He is now with the Edward S. Rogers Sr. Department of Electrical \& Computer Engineering, University of Toronto, Toronto, ON M5S 3G4 Canada. Email: alireza.mohammadi@mail.utoronto.ca

M. Tavakoli is with the Department of Electrical \& Computer Engineering, University of Alberta, Edmonton, AB T6G 2V4 Canada. Email: mahdi.tavakolieualberta.ca

H. J. Marquez is with the Department of Electrical \& Computer Engineering, University of Alberta, Edmonton, AB T6G 2V4 Canada. Email: hmarquez@ualberta.ca reduce the mental load of the human operator as well as the impact forces [1].

Wireless and IP based networks are cheap and pervasive communication media most suitable for long-distance teleoperation systems. For instance, telerehabilitation is a potential teleoperation application that can use the Internet as a communication medium for delivery of physiotherapy services to home-based disabled patients in a fast and convenient way [2]. The variable time delay inherent in the required Internetbased network communication can cause severe instability and performance degradation in the teleoperation system. Some solutions have been proposed to tackle the problems associated with variable time delay. In [3], communication management modules were incorporated into a variable-delay bilateral teleoperation system to reconstruct the scattering variable while ensuring the passivity of the communication block. In [4], a time forward observer was used to ensure the passivity of a teleoperation system with variable time delay when there were no model mismatches and the slave robot did not interact with hard surfaces in the remote environment. These solutions, however, are unable to ensure the stability and position tracking of the teleoperation system [5].

In order to tackle the above mentioned stability and position tracking problems, Nuno et al. developed a general framework based on Lyapunov approach to analyze the stability and performance of nonlinear teleoperation systems with variable time delays [5], [6]. A common shortcoming of this work and other prior art is that they do not consider the effects of disturbances acting on the teleoperation system despite the fact that the master and the slave manipulators are generally subject to effects such as joint frictions and unknown end-effector payloads. Employing disturbance observers can pave the way for suppressing such disturbances [7], [8].

Disturbance observers have been used in several telerobotic applications. A number of these disturbance observer based control schemes can achieve a transparent teleoperation system only in the absence of time delay while there is no guarantee of stability in the presence of communication time delays. In [9], a disturbance observer was used at the slave side of a nonlinear unilateral teleoperation system in order to improve the position tracking between the master and the slave while the slave robot was moving in free space. In [10], the authors implemented a pair of nonlinear disturbance observers in a 4-channel bilateral teleoperation architecture to achieve full transparency in the absence of communication time delays in both free and constrained motions.

In this paper, we will present a novel nonlinear disturbance observer based control law for teleoperation systems with variable time delays. By using a Lyapunov approach similar to Nuno et al., we develop a disturbance observer based control scheme that is able to guarantee asymptotic disturbance 
tracking, asymptotic position tracking, and stability of the teleoperation system in the presence of variable time delays and disturbances.

The organization of this paper is as follows. First, the dynamics of a teleoperation system is presented in Section II. Next, disturbance observer based control laws for variable time delay teleoperation and the main results of this paper are given in Section III. Finally, we demonstrate the effectiveness of the proposed design method developed throughout the paper by performing experiments on a pair of PHANToM Omni ${ }^{\circledR}$ (SensAble Technologies Inc., MA, USA) haptic devices forming a teleoperation system in Section IV.

\section{DYNAMICS OF TELEOPERATION SYSTEMS}

In the remainder of this paper, we will adopt the following notation system:

Notation. We denote the set of real numbers, positive real numbers, and positive real numbers and zero by $R, R^{+}$, and $\mathcal{R}_{0}^{+}$, respectively. We represent the maximum and the minimum eigenvalues of a square matrix by $\lambda_{\max }($.$) and$ $\lambda_{\text {min }}($.$) , respectively. By a vector norm we mean the vector$ 2 -norm and by a matrix norm we mean the induced matrix 2-norm. Thus, $\mathbf{x} \in \mathcal{R}^{n} \Rightarrow|\mathbf{x}|=\sqrt{\mathbf{x}^{T} \mathbf{x}}, \mathbf{X} \in \mathcal{R}^{n \times n} \Rightarrow|\mathbf{X}|=$ $\sqrt{\lambda_{\max }\left(\mathbf{X}^{T} \mathbf{X}\right)}$. We denote the space of signals with bounded energy and the $\mathcal{L}_{2}$ norm of vector signals by $\mathcal{L}_{2}$ and $\|.\|_{2}$, respectively. Therefore, $\mathbf{f}(.) \in \mathcal{L}_{2} \Longleftrightarrow \| \mathbf{f}||_{2}^{2}=\int_{0}^{\infty}|\mathbf{f}(\sigma)|^{2} d \sigma<$ $\infty$. We denote the space of bounded signals and the $\mathcal{L}_{\infty}$ norm of vector signals by $\mathcal{L}_{\infty}$ and $\|.\|_{\infty}$, respectively. Thus, $\mathbf{f}(.) \in \mathcal{L}_{\infty} \Longleftrightarrow|| \mathbf{f}||_{\infty}=\sup _{t>0}|\mathbf{f}(t)|<\infty, \forall t \geq 0 . \diamond$

We consider the master and the slave devices to be rigid $n$-degree of freedom (DOF) serial robotic manipulators. The following dynamic equations govern the motions of the master and the slave manipulators, subject to disturbances, in the joint space [11]:

$$
\begin{aligned}
& \hat{\mathbf{M}}_{m}\left(\mathbf{q}_{m}\right) \ddot{\mathbf{q}}_{m}+\hat{\mathbf{C}}_{m}\left(\mathbf{q}_{m}, \dot{\mathbf{q}}_{m}\right) \dot{\mathbf{q}}_{m}+\hat{\mathbf{G}}_{m}\left(\mathbf{q}_{m}\right)=\boldsymbol{\tau}_{c m} \\
& -\boldsymbol{\tau}_{h}+\boldsymbol{\tau}_{d m}^{*} \\
& \hat{\mathbf{M}}_{s}\left(\mathbf{q}_{s}\right) \ddot{\mathbf{q}}_{s}+\hat{\mathbf{C}}_{s}\left(\mathbf{q}_{s}, \dot{\mathbf{q}}_{s}\right) \dot{\mathbf{q}}_{s}+\hat{\mathbf{G}}_{s}\left(\mathbf{q}_{s}\right)=-\boldsymbol{\tau}_{c s} \\
& +\boldsymbol{\tau}_{e}+\boldsymbol{\tau}_{d s}^{*}
\end{aligned}
$$

where $\mathbf{q}_{i}, \dot{\mathbf{q}}_{i}, \ddot{\mathbf{q}}_{i} \in R^{n \times 1}, i=m, s$ are the vectors of joint positions, velocities, and accelerations of the master and the slave, respectively. Also, $\hat{\mathbf{M}}_{i}\left(\mathbf{q}_{i}\right), i=m, s$ are the symmetric and positive definite estimates of the inertia matrices of the master and the slave (see Remark 1), and satisfy ${ }^{1}$

$$
\begin{aligned}
\mu_{m} \mathbf{I} \leq \hat{\mathbf{M}}_{m}\left(\mathbf{q}_{m}\right) & \leq \mu_{M} \mathbf{I} \\
\mu_{s} \mathbf{I} \leq \hat{\mathbf{M}}_{s}\left(\mathbf{q}_{s}\right) & \leq \mu_{S} \mathbf{I} .
\end{aligned}
$$

where $\mu_{i}$ 's are positive constants. Similarly, $\hat{\mathbf{C}}_{i}\left(\mathbf{q}_{i}, \dot{\mathbf{q}}_{i}\right)$ and $\hat{\mathbf{G}}_{i}\left(\mathbf{q}_{i}\right)$ represent the centrifugal/Coriolis matrix and the gravity vector estimates (see Remark 1 below for choosing these estimates), respectively. The centrifugal/Coriolis matrix estimates in this paper are chosen such that $\dot{\hat{\mathbf{M}}}_{i}\left(\mathbf{q}_{i}\right)-2 \hat{\mathbf{C}}_{i}\left(\mathbf{q}_{i}, \dot{\mathbf{q}}_{i}\right)$ is skew-symmetric. We have

\footnotetext{
${ }^{1}$ By $\mathbf{A} \geq \mathbf{B}$, where $\mathbf{A}$ and $\mathbf{B}$ are square matrices we mean that $\mathbf{A}-\mathbf{B}$ is a positive semi-definite matrix.
}

$$
\begin{array}{r}
{\left[\dot{\hat{\mathbf{M}}}_{i}\left(\mathbf{q}_{i}\right)-2 \hat{\mathbf{C}}_{i}\left(\mathbf{q}_{i}, \dot{\mathbf{q}}_{i}\right)\right]^{T}=-\left[\dot{\hat{\mathbf{M}}}_{i}\left(\mathbf{q}_{i}\right)-2 \hat{\mathbf{C}}_{i}\left(\mathbf{q}_{i}, \dot{\mathbf{q}}_{i}\right)\right]} \\
\Rightarrow \dot{\hat{\mathbf{M}}}_{i}\left(\mathbf{q}_{i}\right)=\hat{\mathbf{C}}_{i}\left(\mathbf{q}_{i}, \dot{\mathbf{q}}_{i}\right)+\hat{\mathbf{C}}^{T}\left(\mathbf{q}_{i}, \dot{\mathbf{q}}_{i}\right) .
\end{array}
$$

Moreover, $-\tau_{h}$ and $\tau_{e}$ are the torques exerted by the human operator and the remote environment, and $\boldsymbol{\tau}_{c m}$ and $\boldsymbol{\tau}_{c s}$ are the control torques applied to the master and the slave, respectively.

The disturbance torques $\tau_{d m}^{*}$ and $\tau_{d s}^{*}$ lump the effects of all dynamic uncertainties, joint frictions, and external disturbances such as end-effector payload exerted to the master and the slave, respectively. We have

$$
\boldsymbol{\tau}_{d i}^{*}=\boldsymbol{\tau}_{\text {ext }, i}-\Delta \mathbf{M}_{i} \ddot{\mathbf{q}}_{i}-\Delta \mathbf{C}_{i} \dot{\mathbf{q}}_{i}-\Delta \mathbf{G}_{i}-\mathbf{F}_{i}\left(\dot{\mathbf{q}}_{i}\right), i=m, s
$$

where $\tau_{\text {ext }, i}, \Delta \mathbf{M}_{i}, \Delta \mathbf{C}_{i}, \Delta \mathbf{G}_{i}, \mathbf{F}_{i}\left(\dot{\mathbf{q}}_{i}\right)$ represent the external disturbance, the manipulator model uncertainties, and the joint friction vector, respectively. Next, by incorporating the human operator and the remote environment torques into $\tau_{d m}^{*}$ and $\tau_{d s}^{*}$, we define the following lumped disturbance vectors:

$$
\begin{gathered}
\tau_{d m}=\boldsymbol{\tau}_{d m}^{*}-\boldsymbol{\tau}_{h} \\
\tau_{d s}=\boldsymbol{\tau}_{d s}^{*}+\boldsymbol{\tau}_{e}
\end{gathered}
$$

Incorporating the human operator and the remote environment torques into the lumped disturbance terms (7)-(8) enable us to guarantee the position tracking between the master and the slave manipulators in both free and constrained motions without the need for additional force/torque sensors.

Remark 1. If the exact model of the master and the slave manipulators are not available, then the approximate inertia matrices $\hat{\mathbf{M}}_{i}\left(\mathbf{q}_{i}\right)$ and $\hat{\mathbf{C}}_{i}\left(\mathbf{q}_{i}, \dot{\mathbf{q}}_{i}\right), i=m, s$, can be chosen to be any arbitrary matrices satisfying (3)-(5), in implementing our control law and disturbance observers. For instance, $\hat{\mathbf{M}}_{i}\left(\mathbf{q}_{i}\right)$, can be a constant, positive-definite and symmetric matrix in which case $\hat{\mathbf{C}}_{i}\left(\mathbf{q}_{i}, \dot{\mathbf{q}}_{i}\right)$ will be chosen to be zero according to (5). As another example, the estimated Denavit-Hartenberg (D-H) parameters of a robotic manipulator may be used to find the estimate of its inertia and centrifugal/Coriolis matrices because such choices are guaranteed to meet (3)(5). The gravity vector of the robotic manipulator can also be estimated using a constant vector or approximate D-H parameters of the robotic manipulator. $\diamond$

Figure 1 shows the block diagram of a teleoperation system subject to disturbances and variable communication time delays with disturbance observers and controllers incorporated into it. As it can be seen in Figure 1, the teleoperation system consists of five subsystems, namely, the human operator, the master and its associated controller and disturbance observer, the communication channel with variable time delays $T_{i}(t), i=m, s$, from the master to the slave and vice versa, the slave and its associated controller and disturbance observer, and the remote environment.

Assumptions. We will assume that the human operator and the remote environment are passive systems. This is a common assumption that is frequently encountered in the teleoperation literature and is the basis of passivitybased control of teleoperation systems with time delay [12], [13]. This assumption enables us to analyze the stability 
and performance of a teleoperation system independent of the dynamic models of the human operator and the remote environment [13]. The assumption of a passive environment is reasonable as most environments do not generate energy. Also, there is ample research that shows that the human arm displays passive dynamics while it maintains stable contact with any strictly passive object (see, for example, [14] and [15]) despite active control by the central nervous system [16]. Having considered the human operator and the remote environment as passive velocity to torque/force mappings, we have

$$
\begin{gathered}
\exists v_{m}>0 \text { such that } \int_{0}^{t} \dot{\mathbf{q}}_{m}^{T}(\sigma) \tau_{h}(\sigma) d \sigma \geq-v_{m}, \forall t \geq 0 \\
\exists v_{s}>0 \text { such that }-\int_{0}^{t} \dot{\mathbf{q}}_{s}^{T}(\sigma) \tau_{e}(\sigma) d \sigma \geq-v_{s}, \forall t \geq 0 .
\end{gathered}
$$

Also, we make the following assumptions about the variable time delay in the communication channel:

- There exist a known upper bound on the variable time delay:

$$
0 \leq T_{i}(t) \leq T_{\text {max } \_i}<\infty, \forall t \geq 0 \text { and } i=m, s .
$$

- The rate of change of the variable time delay is bounded:

$$
\left|\dot{T}_{i}(t)\right| \leq \zeta_{T_{-} i}<\infty, \forall t \geq 0 \text { and } i=m, s .
$$

Remark 2. The assumptions (11)-(12) are frequently encountered in network-based control (see, for example, [6], [17]). Also, note that we do not need to know the value of $\zeta_{T_{-} i}$ to design the controllers. $\diamond$

\section{DISTURBANCE OBSERVER BASED CONTROL LAWS}

In this section, we present our disturbance observer based control laws. We extend past work of [5] on variable time delay teleoperation by considering the disturbances acting on the master and the slave robotic manipulators. Our stability and performance goals are:

- Ensuring boundedness of the master and the slave position trajectories, in both free and constrained motions (when the master and the slave are in contact with the operator and the remote environment) independent of the variable time delays present in the communication channel and in the presence of disturbances,

- Synchronizing the positions of the joints of the master and the slave manipulators in both free and constrained motions. Joint-level position synchronization between master and slave has applications such as arm/hand teleoperation where the human operator wears a data glove to control the dexterous hand of the slave robot [18].

\section{A. Synchronizing control torques}

We will apply the following control torques to the master and the slave robotic manipulators:

$$
\begin{aligned}
\boldsymbol{\tau}_{c m} & =\hat{\mathbf{G}}_{m}\left(\mathbf{q}_{m}\right)-\hat{\boldsymbol{\tau}}_{d m}+\boldsymbol{\tau}_{m} \\
\boldsymbol{\tau}_{c s} & =-\hat{\mathbf{G}}_{s}\left(\mathbf{q}_{s}\right)+\hat{\boldsymbol{\tau}}_{d s}+\boldsymbol{\tau}_{s}
\end{aligned}
$$

where $\hat{\boldsymbol{\tau}}_{d m}$ and $\hat{\boldsymbol{\tau}}_{d s}$ are the estimates of the master and the slave disturbances provided by the disturbance observers introduced later in this section. Also, $\boldsymbol{\tau}_{m}$ and $\boldsymbol{\tau}_{s}$ are the following proportional-damping synchronizing torques applied to the master and the slave, respectively [6], [5]. We have

$$
\begin{aligned}
\boldsymbol{\tau}_{m} & =k_{m}\left[\mathbf{q}_{s}\left(t-T_{s}(t)\right)-\mathbf{q}_{m}\right]-b_{m} \dot{\mathbf{q}}_{m} \\
\boldsymbol{\tau}_{s} & =k_{s}\left[\mathbf{q}_{s}-\mathbf{q}_{m}\left(t-T_{m}(t)\right)\right]+b_{s} \dot{\mathbf{q}}_{s}
\end{aligned}
$$

where $\mathbf{q}_{m}\left(t-T_{m}(t)\right)$ and $\mathbf{q}_{s}\left(t-T_{s}(t)\right)$ are the delayed joint positions of the master and the slave that have been transmitted through the communication channel (see Figure 1). The parameters $k_{i}, b_{i}, i=m, s$ are positive and real constants to be determined.

Remark 3. There are two other synchronizing control torques, namely, a PD-like and a scattering-based controller for teleoperation systems with variable time delays [5]. However, the rate of change of time delays needs to be known in these two schemes and is required to be less than unity, which is not the case in packet switched communication networks, such as the Internet. Moreover, as shown by [5], these two schemes result in an inferior performance in comparison with the proportional-damping scheme of (15)-(16). Therefore, we will only address the proportionaldamping scheme and use it in the disturbance observer based control of teleoperation system of (1)-(2) with variable time delays. $\diamond$

Applying the control torques (13)-(14) to the teleoperation system (1)-(2) yields the following closed-loop equations:

$$
\begin{aligned}
& \hat{\mathbf{M}}_{m}\left(\mathbf{q}_{m}\right) \ddot{\mathbf{q}}_{m}+\hat{\mathbf{C}}_{m}\left(\mathbf{q}_{m}, \dot{\mathbf{q}}_{m}\right) \dot{\mathbf{q}}_{m}=\boldsymbol{\tau}_{m}+\Delta \boldsymbol{\tau}_{d m} \\
& \hat{\mathbf{M}}_{s}\left(\mathbf{q}_{s}\right) \ddot{\mathbf{q}}_{s}+\hat{\mathbf{C}}_{s}\left(\mathbf{q}_{s}, \dot{\mathbf{q}}_{s}\right) \dot{\mathbf{q}}_{s}=-\boldsymbol{\tau}_{s}+\Delta \boldsymbol{\tau}_{d s}
\end{aligned}
$$

where $\Delta \boldsymbol{\tau}_{d i}=\boldsymbol{\tau}_{d i}-\hat{\boldsymbol{\tau}}_{d i}, i=m, s$ is the disturbance tracking error. Determining the disturbance estimates $\hat{\boldsymbol{\tau}}_{d m}$ and $\hat{\boldsymbol{\tau}}_{d s}$ will be addressed later in this section. Note that if we have perfect disturbance tracking, i.e., $\Delta \boldsymbol{\tau}_{d i}=0, i=m, s$, disturbances will be canceled out in the closed-loop equation of the teleoperation system and it seems as if we are dealing with robotic manipulators with known dynamics and in free motion.

Now, we will present a lemma for teleoperation systems in free motion.

Lemma (Teleoperator's Zero-convergence Lemmas). [19] Consider the teleoperation system subject to disturbances as described by (17)-(18), and in free motion (i.e., with $\boldsymbol{\tau}_{h}=\boldsymbol{\tau}_{e}=\mathbf{0}$ ). We have

- Velocity Zero-convergence. Assume that $\dot{\mathbf{q}}_{i} \in \mathcal{L}_{2} \cap \mathcal{L}_{\infty}$ and $\tau_{i} \in \mathcal{L}_{\infty}$, and

$\Delta \tau_{d i} \in \mathcal{L}_{\infty}$ for $i=m, s$. Then, $\lim _{t \rightarrow \infty}\left|\dot{\mathbf{q}}_{i}\right|=0$.

- Acceleration Zero-convergence. In addition to the previous assumptions, assume that $\dot{\boldsymbol{\tau}}_{i} \in \mathcal{L}_{\infty}$, and $\Delta \dot{\boldsymbol{\tau}}_{d i} \in$ $\mathcal{L}_{\infty}$ for $i=m, s$. Then, $\ddot{\mathbf{q}}_{i}$ is uniformly continuous and $\lim _{t \rightarrow \infty}\left|\ddot{\mathbf{q}}_{i}\right|=0$.

\section{B. Disturbance observers}

Inspired by the nonlinear disturbance observers proposed by [7], [8], we propose the following disturbance observer to be employed at the master side of the teleoperation system: 


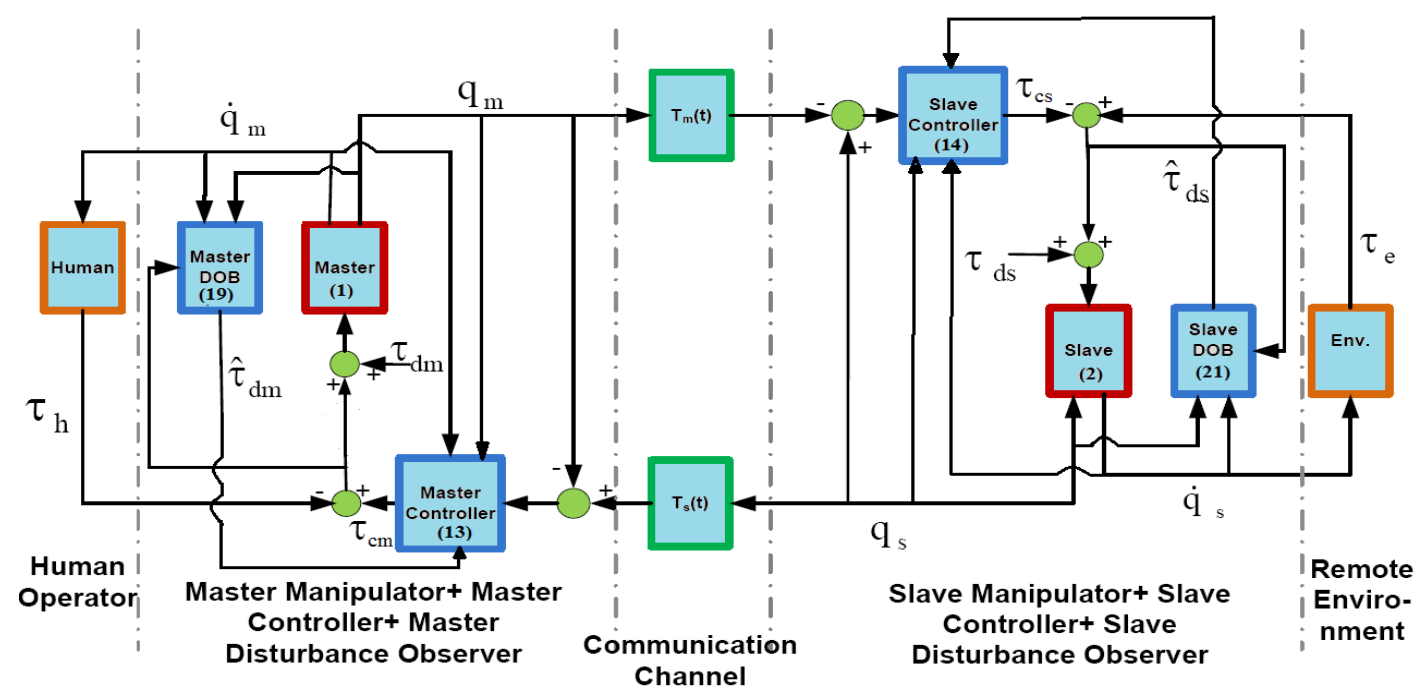

Fig. 1. Disturbance observer based control of a teleoperation system with variable time delays.

$$
\begin{aligned}
& \dot{\mathbf{z}}_{m}=-\alpha_{m} \hat{\mathbf{M}}_{m}^{-1}\left(\mathbf{q}_{m}\right) \mathbf{z}_{m}+\alpha_{m} \hat{\mathbf{M}}_{m}^{-1}\left(\mathbf{q}_{m}\right)[ \\
& \hat{\mathbf{C}}_{m}\left(\mathbf{q}_{m}, \dot{\mathbf{q}}_{m}\right) \dot{\mathbf{q}}_{m}+\hat{\mathbf{G}}_{m}\left(\mathbf{q}_{m}\right) \\
& \left.-\boldsymbol{\tau}_{c m}-\alpha_{m} \dot{\mathbf{q}}_{m}\right]+\dot{\mathbf{q}}_{m} \\
& \hat{\boldsymbol{\tau}}_{d m}=\mathbf{z}_{m}+\alpha_{m} \dot{\mathbf{q}}_{m}
\end{aligned}
$$

where $\alpha_{m}$ is a positive real constant.

Remark 4. The last term $\dot{\mathbf{q}}_{m}$ in (19) do not exist in the nonlinear disturbance observer proposed in [7], [8]. These new terms will help us to achieve desired stability and performance in the proposed control scheme. $\diamond$

Here, we will assume that the rate of change of the lumped disturbances are negligible in comparison with the dynamics of the disturbance observer. The dynamics of the master-side disturbance tracking error according to (1), (13), (17), and (19) is:

$$
\Delta \dot{\boldsymbol{\tau}}_{d m}=-\dot{\mathbf{q}}_{m}-\alpha_{m} \hat{\mathbf{M}}_{m}^{-1}\left(\mathbf{q}_{m}\right) \Delta \boldsymbol{\tau}_{d m} .
$$

Similar to the disturbance observer at the master side, we employ the following disturbance observer at the slave side of the teleoperation system:

$$
\begin{aligned}
\dot{\mathbf{z}}_{s}= & -\alpha_{s} \hat{\mathbf{M}}_{s}^{-1}\left(\mathbf{q}_{s}\right) \mathbf{z}_{s}+\alpha_{s} \hat{\mathbf{M}}_{s}^{-1}\left(\mathbf{q}_{s}\right)\left[\hat{\mathbf{C}}_{s}\left(\mathbf{q}_{s}, \dot{\mathbf{q}}_{s}\right) \dot{\mathbf{q}}_{s}+\right. \\
& \left.\hat{\mathbf{G}}_{s}\left(\mathbf{q}_{s}\right)+\boldsymbol{\tau}_{c s}-\alpha_{s} \dot{\mathbf{q}}_{s}\right]+\dot{\mathbf{q}}_{s} \\
\hat{\boldsymbol{\tau}}_{d s}= & \mathbf{z}_{s}+\alpha_{s} \dot{\mathbf{q}}_{s}
\end{aligned}
$$

where $\alpha_{s}$ is a positive real constant. The dynamics of the slave-side disturbance tracking error will be

$$
\Delta \dot{\boldsymbol{\tau}}_{d s}=-\dot{\mathbf{q}}_{s}-\alpha_{s} \hat{\mathbf{M}}_{s}^{-1}\left(\mathbf{q}_{s}\right) \Delta \boldsymbol{\tau}_{d s}
$$

\section{Stability and performance analysis}

Now, we are ready to state the main result of this paper in the form of the following theorem.

Theorem 1: Consider the teleoperation system described by (1)-(2). This teleoperation system is subject to variable time delays satisfying (11) and (12). The master and the slave disturbance observers are given in (19) and (21). The disturbance observer based control laws are given in (13) and (14) with the synchronization torques $\tau_{m}$ and $\boldsymbol{\tau}_{s}$ given in (15) and (16). Assume that the disturbance observer gains and the controller gains satisfy $\alpha_{i}>0, k_{i}>0, b_{i}>0$, for $i=m, s$. Also, assume that the controller gains are set such that the following inequality is satisfied:

$$
4 b_{m} b_{s}>\left(T_{\max \lrcorner m}^{2}+T_{\text {max } s}^{2}\right) k_{m} k_{s} .
$$

Then:

- The velocities and position error are bounded, i.e., $\left\{\dot{\mathbf{q}}_{i}, \mathbf{q}_{m}-\mathbf{q}_{s}\right\} \in \mathcal{L}_{\infty}$ and $\dot{\mathbf{q}}_{i} \in \mathcal{L}_{2}$. Moreover, $\left\{\mathbf{q}_{m}-\mathbf{q}_{s}(t-\right.$ $\left.\left.T_{s}(t)\right), \mathbf{q}_{s}-\mathbf{q}_{m}\left(t-T_{m}(t)\right)\right\} \in \mathcal{L}_{\infty}$. Also, there is asymptotic disturbance tracking, i.e., $\lim _{t \rightarrow \infty} \Delta \tau_{d i}(t)=0$.

- Accelerations and velocities asymptotically converge to zero and asymptotic position tracking is achieved, i.e., $\lim _{t \rightarrow \infty}\left|\mathbf{q}_{m}-\mathbf{q}_{s}\left(t-T_{s}(t)\right)\right|=\lim _{t \rightarrow \infty}\left|\mathbf{q}_{s}-\mathbf{q}_{m}\left(t-T_{m}(t)\right)\right|=0$.

Proof: The proof has been omitted due to page limits and can be found in Theorem 4.1 in [20].

Inequality (23) gives a sufficient condition for the parameters of synchronizing control torques (15)-(16) to ensure the stability of the teleoperation system in the presence of variable time delays and disturbances. Figure 2 depicts the stability region of the teleoperation system with known maximum time delays $T_{\max -i}, i=m, s$. Note that we have taken a conservative approach towards stabilizing the teleoperation system by considering maximum time delays instead of their statistical properties. Hence, there might be points in the potentially unstable region of Figure 2 at which the teleoperation system is still stable.

Remark 5. The inequality (23) indicates a trade off between the controller damping gains, the controller proportional gains and the maximum time delays in the communication channel between the master and the slave. The larger the maximum communication time delays, the larger the damping gains or the smaller the proportional gains need to be to preserve the stability of the teleoperation system. 


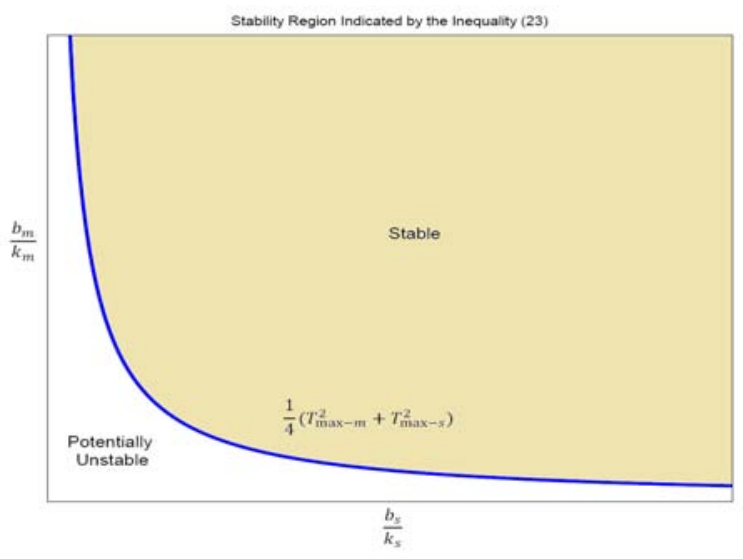

Fig. 2. Stability region of the teleoperation system indicated by Inequality (23).

However, larger damping gains or smaller proportional gains result in more damped and slower responses and thus inferior teleoperation performance. Therefore, given maximum communication time delays, (23) provides practically useful guidelines in terms of choosing the minimum possible damping gains and maximum possible proportional gains to get the best possible performance while guaranteeing stability. $\diamond$

\section{EXPERIMENTS}

In this section, experiments are carried out using PHANToM Omni ${ }^{\circledR}$ haptic devices in order to demonstrate the effectiveness of the proposed disturbance observer based bilateral teleoperation scheme.

\section{A. Experimental setup}

The PHANToM Omni is a haptic device that can be used for a variety of purposes including virtual reality and teleoperation applications. The PHANToM Omni has three actuated revolute joints that provide the user with force feedback. In addition to the actuated joints, the PHANToM haptic has 3 wrist joints that are passive. In these experiments, we will use the first three actuated joints of the PHANToM device. We use PHANSIM Toolkit, a Simulink toolkit for the control of SenSable haptic devices, in our experiments [21].

Two PHANToM haptic devices are connected to a computer in a daisy chain configuration. The PHANToM Omnis end-effector position and orientation data are collected at a frequency of $1000 \mathrm{~Hz}$. In the experiments, the human operator moves the master haptic device, causing slave haptic device that is constrained by a rubber band to a stiff wall to move.

The one-way variable time delay in the communication channel is generated by a random process with shifted gamma distribution. This distribution has been used to model the Internet-based communication time delays [22], [23]. The probability density function of shifted gamma is:

$$
f(x)=\frac{\left(\frac{x-\gamma}{\beta}\right)^{\alpha-1} \cdot \exp \left(-\frac{x-\gamma}{\beta}\right)}{\beta \cdot \Gamma(\alpha)} .
$$

We choose $\alpha=0.85, \beta=0.09$, and $\gamma=0.085$ in the experiments. These time delays are shown in Figure 3. As it can be seen, the maximum time delay is $0.7^{\mathrm{sec}}$.
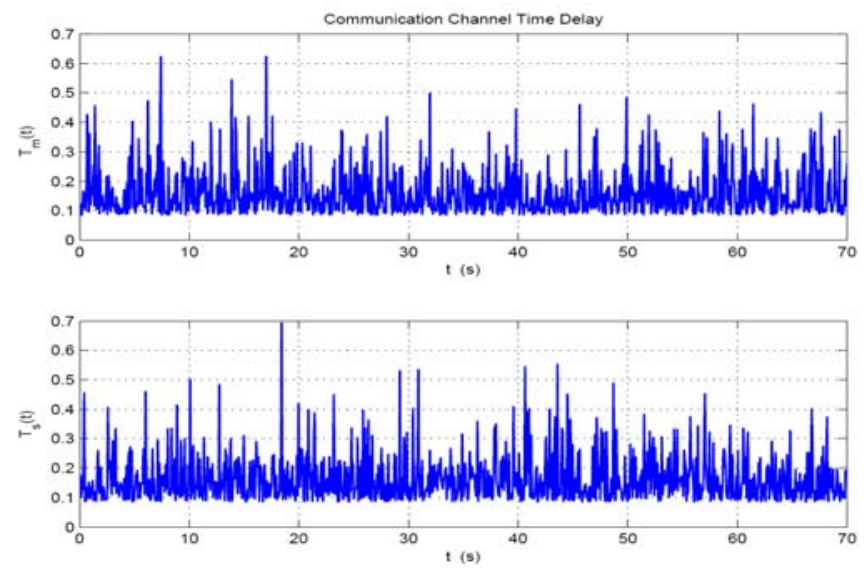

Fig. 3. Communication channel time delay in the experiment.

The parameters of the synchronizing torques (control laws), given in (15) and (16), are considered to be $k_{i}=0.5$ and $b_{i}=$ $0.25, i=m, s$. Note that these gains satisfy the inequality (23) of Theorem 1. The teleoperation control torques are given by (13) and (14). The mass matrices, Coriolis/centrifugal matrices, and the gravity vectors of the haptic devices are estimated by $\hat{\mathbf{M}}_{i}\left(\mathbf{q}_{i}\right)=0.25 \mathbf{I}, \hat{\mathbf{C}}_{i}\left(\mathbf{q}_{i}, \dot{\mathbf{q}}_{i}\right)=0, \hat{\mathbf{G}}_{i}\left(\mathbf{q}_{i}\right)=0$. Here, the human operator and the remote environment exerted torques, the gravity forces that are acting on the second and the third joints of the haptic devices, and the friction torques acting on the joints of the haptic devices are the disturbances.

\section{B. Experimental results}

The human operator moves the master arm in 3 dimensional space from $t=0 \mathrm{sec}$ to $t=60 \mathrm{sec}$, while the slave arm is connected to the rubber band. At $t=61 \mathrm{sec}$ the human operator moves the first joint of the master arm quickly and releases it in order to test the stability of the teleoperation system. Figures 4 and 5 depict the position tracking response of the teleoperation system when no disturbance observers are employed in the teleoperation system. The gravity forces acting on the second and the third joints of the haptic device, and the exerted torque by the rubber band to the slave haptic device cause poor tracking response of the teleoperation system. In Figure 5, we increased the proportional gain of the controller to $k=0.65$ in order to improve the tracking performance of the teleoperation system without disturbance observers. As it can be observed, the tracking response of the teleoperation system has been slightly improved. On the other hand, this proportional gain violates the stability condition of (23). Therefore, the teleoperation system becomes unstable at $t=61 \mathrm{sec}$.

Figure 6 depicts the position tracking response of the teleoperation system when disturbance observers are employed in the teleoperation system with proportional gain $k=0.5$ and damping gain $b=0.25$. As it can be observed from the figure, the slave haptic device closely follows the master haptic device. Also, the teleoperation system maintains its stability after the operator tries to destabilize the system.

\section{CONCLUSION}

In this paper, a novel nonlinear disturbance observer based control scheme for bilateral teleoperation systems with variable time delays is introduced. It is shown that bilateral 

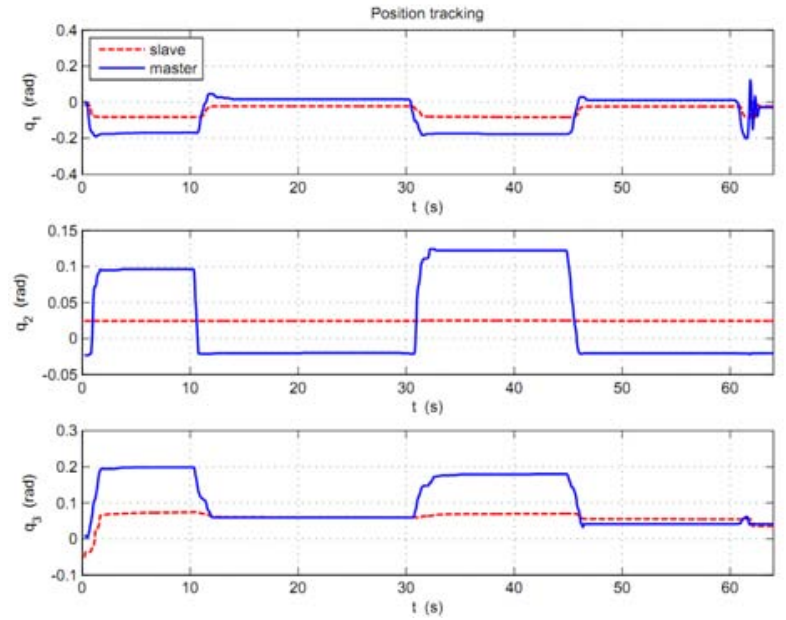

Fig. 4. Experiment : Position tracking response of the teleoperation system without disturbance observers $\left(k_{i}=0.5, b_{i}=0.25, \mathrm{i}=\mathrm{m}, \mathrm{s}\right)$.
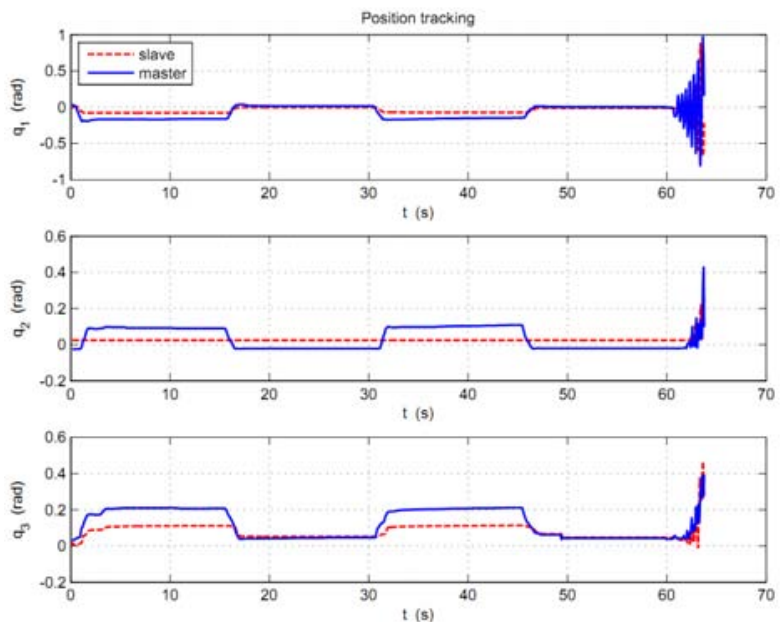

Fig. 5. Experiment : Position tracking response of the teleoperation system without disturbance observers and high proportional gain $\left(k_{i}=0.65, b_{i}=\right.$ $0.25, \mathrm{i}=\mathrm{m}, \mathrm{s})$.

teleoperation systems can be stabilized in the presence of disturbances and variable communication time delays. Also, asymptotic disturbance and position tracking can be achieved in both free and constrained motions. Simulations and experiments are performed to further demonstrate the efficiency of the proposed disturbance observer based teleoperation control scheme.

\section{REFERENCES}

[1] G. A. V. Christiansson, "An experimental study of haptic feedback in a teleoperated assembly task," J. Comput. Inf. Sci. Eng., vol. 8, no. 4, Dec. 2008.

[2] H. S. Park, Q. Peng, and L. Q. Zhang, "A portable telerehabilitation system for remote evaluations of impaired elbows in neurological disorders," IEEE Trans. Neur. Syst. Reh., vol. 16, no. 3, pp. 245-254, Jun. 2008.

[3] N. Chopra, P. Berestesky, and M. W. Spong, "Bilateral teleoperation over unreliable communication networks," IEEE Trans. Contr. Syst. Technol., vol. 16, no. 2, pp. 304-313, Mar. 2008.

[4] S. Munir and W. Book, "Control techniques and programming issues for time delayed internet based teleoperation," ASME J. Dyn. Syst. Meas. Contr., vol. 125, no. 2, pp. 205-214, Jun. 2003.

[5] E. Nuno, L. Basanez, R. Ortega, and M. W. Spong, "Position tracking for non-linear teleoperators with variable time delay," Int. J. Robot. Res., vol. 28, no. 7, pp. 895-910, Jul. 2009.
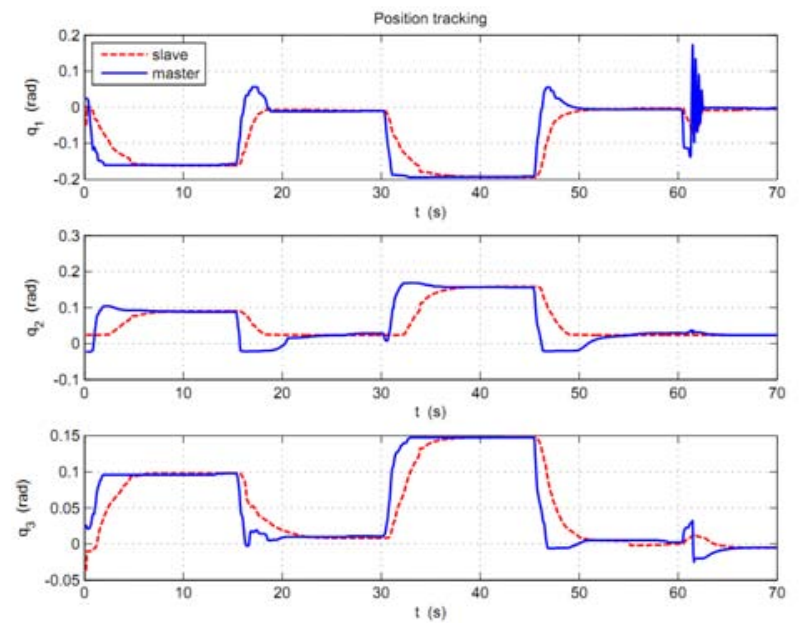

Fig. 6. Experiment : Position tracking response of the teleoperation system with disturbance observers $\left(k_{i}=0.5, b_{i}=0.25, \mathrm{i}=\mathrm{m}, \mathrm{s}\right)$.

[6] E. Nuno, L. Basanez, and R. Ortega, "Passivity based control for bilateral teleoperation: A tutorial," Automatica, vol. 47, pp. 485-495, Feb. 2011.

[7] W. H. Chen, D. J. Ballance, P. J. Gawthrop, and J. O'Reilly, “A nonlinear disturbance observer for robotic manipulators," IEEE Trans. Ind. Electron., vol. 47, no. 4, pp. 932-938, Aug. 2000.

[8] A. Nikoobin and R. Haghighi, "Lyapunov-based nonlinear disturbance observer for serial n-link robot manipulators," J. Intell. Robot. Syst., vol. 55, no. 2-3, pp. 135-153, Jul. 2009.

[9] S. Lichiardopol, N. van de Wouw, D. Kostic, and H. Nijmeijer, "Trajectory tracking control for a tele-operation system setup with disturbance estimation and compensation," in Proc. IEEE Conf. Decision and Control, 2010, pp. 1142-1147.

[10] A. Mohammadi, M. Tavakoli, and H. J. Marquez, "Disturbance observer based control of nonlinear haptic teleoperation systems," IET Control Theory Appl., vol. 5, no. 17, pp. 2063-2074, 2011.

[11] M. W. Spong, S. Hutchinson, and M. Vidyasagar, Robot Modeling and Control. New York: Wiley, 2005.

[12] P. Hokayem and M. W. Spong, "Bilateral teleoperation: An historical survey," Automatica, vol. 42, pp. 2035-2057, 2006.

[13] G. Niemeyer, C. Preusche, and G. Hirzinger, "Telerobotics," in Springer Handbook of Robotics, B. Siciliano and O. Khatib, Eds. Springer-Verlag, 2008, pp. 741-757.

[14] J. E. Speich, L. Shao, and M. Goldfarb, "Modeling the human hand as it interacts with a telemanipulation system," Mechatronics, vol. 15, no. 9, pp. 1127-1142, 2005.

[15] J. Colgate and N. Hogan, "Robust control of dynamically interacting systems," Int. J. Control, vol. 48, no. 1, pp. 65-88, 1988.

[16] N. Hogan, "Controlling impedance at the man/machine interface," in Proc. IEEE. Int. Conf. Robot. Autom., 1989, pp. 1621-1631.

[17] H. Gao, T. Chen, and J. Lam, "A new delay system approach to network-based control," Automatica, vol. 44, pp. 39-52, 2008.

[18] H. Hu, J. Li, Z. Xie, B. Wang, H. Liu, and G. Hirzinger, "A robot arm/hand teleoperation system with telepresence and shared control," in Proc. IEEE/ASME Int. Conf. Adv. Intell. Mechatron., 2005, pp. 1312-1317.

[19] E. Nuno, L. Basanez, and R. Ortega, "Control of teleoperators with time-delay: a lyapunov approach," in Topics in Time Delay Systems, J. J. Loiseau, W. Michiels, N. S.-I., and R. Sipahi, Eds. SpringerVerlag, 2009, pp. 371-381.

[20] A. Mohammadi, "Disturbance observer design for robotic and telerobotic systems," Master's thesis, University of Alberta, Edmonton, $\mathrm{AB}, 2011$.

[21] A. Mohammadi, M. Tavakoli, and A. Jazayeri, "Phansim: A simulink toolkit for the sensable haptic devices," in Proc. Canadian Congress of Applied Mechanics, Vancouver, BC, Jun. 2011, pp. 787-790.

[22] D. Chen, X. Fu, W. Ding, H. Li, N. Xi, and Y. Wang, "Shifted gamma distribution and long-range prediction of roundtrip timedelay for internet-based teleoperation," in Proc. IEEE Int. Conf. Robotics and Biomimetics, 2008, pp. 1261-1266.

[23] S. Kim, J. Y. Lee, and D. K. Sung, "A shifted gamma distribution model for long-range dependent internet traffic," IEEE Commun. Lett., vol. 7, no. 3, pp. 124-126, Mar. 2003. 